\title{
Oral contraceptives induced hepatotoxicity
}

\author{
B. Akshaya Srikanth ${ }^{\mathrm{a}, *}$, V. Manisree ${ }^{\mathrm{b}}$
}

\begin{abstract}
${ }^{a}$ Clinical Pharmacist, Rajiv Gandhi Institute of Medical Sciences, Kadapa, Andhra Pradesh, India

${ }^{\mathrm{b}}$ Department of Obstetrics and Gynecology, Rajiv Gandhi Institute of Medical Sciences, Kadapa, Andhra Pradesh, India
\end{abstract}

Received: 4 November 2012

Accepted: 11 December 2012

\author{
*Correspondence to: \\ Dr. B. Akshaya Srikanth, Pharm.D \\ Email: akshaypharmd@gmail.com
}

\begin{abstract}
Oral Contraceptives are the pharmacological agents used to prevent pregnancy. These are divided as the combined and progestogen methods and are administered orally, transdermally, systemically and via vaginal route. All these methods contain both oestrogen and progestogen. Vigorous usage of oral contraceptives and anabolic steroids as associated with cholestasis, vascular lesions and hepatic neoplasm. Benign hepatic neoplasms are clearly associated with oral contraceptives. In this article we discuss the various hepatocellular complications like cholestasis, benign neoplasm and hepatocellular carcinoma occurred by oral contraceptives.
\end{abstract}

Keywords: Oral contraceptives, Hepatotoxicity, Drug toxicity, Combined oral contraceptive pills, hepatic carcinoma, India

\section{INTRODUCTION}

Hepatotoxicity is caused by the xenobiotics that include prescribed and nonprescribed therapeutic agents including herbal medications, and vast array of other organic and inorganic substances that may be ingested deliberately or accidentally. ${ }^{1}$ Drug induced liver injury can be defined as a liver injury by drugs or herbal medicines leading to liver test abnormalities or liver dysfunction with reasonable exclusion of other etiologies. ${ }^{2}$ More than 900 drugs have been implicated in causing liver injury, chemicals often cause subclinical injury to the liver which manifests only as abnormal liver enzyme tests. Drug induced liver injury is responsible for $5 \%$ of all hospital admissions and $50 \%$ of all acute liver failures. ${ }^{3}$

Drug-induced liver injuries have an acute onset, develop while the medication is being administered, and manifest histological pattern that can be categorized as hepatocellular, cholestatic and mixed., ${ }^{4,5}$ Injury to the liver is largely defined by the increase in the blood level of proteins that are liberated from the damaged hepatotocytes like alanine aminotransferase (ALT). The low level of the plasma proteins such as albumin and clotting factors synthesized by the liver or clinicopathologic evidence of impaired bile flow called cholestasis. For defining of the drug-induced liver disease requires histopathologic characterization rather than syndromic recognition of the liver test abnormalities. ${ }^{6}$
Contraception is defined as the prevention of conception, but generally it is understood to mean the intentional prevention of pregnancy. Oral Contraceptives are the pharmacological agents used to prevent pregnancy. Despite the high contraceptive prevalence, unintended pregnancy is common. About $30 \%$ of pregnancies which end in childbirth are unplanned when they are conceived. ${ }^{7}$

\section{HORMONAL CONTRACEPTION}

Hormonal methods of contraception can be divided into combined and progestogen-only methods.

\section{Combined hormonal contraception}

These are administered orally, transdermally, systemically and via the vaginal route. All the methods contain both oestrogen and progestogen. The oral dose of oestrogen in the combined oral contraceptive pills (COCP) varies from 50 to $15 \mu \mathrm{g}$ and some contains mestranol. Most women now use the so-called low-dose pills containing 30-35 $\mu$ g. Lower dose pills are potentially safer since the cardiovascular risks of the pills are mainly due to oestrogen. The newest COCP in Indian market (Yasmin) contains a progestogen with both anti-androgenic and anti-mineralocarticoid activity (drospirenone). The pill is taken for 21 days followed by a 7-day break when withdrawal bleeding usually occurs. The routine use of the contraceptives associated with the increase of venous thrombosis. Increasing the use of pills together for three months (tricycling) or more likely associated with 


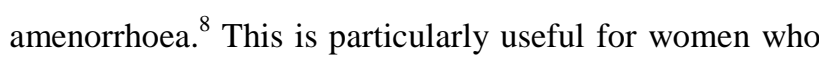
experience symptoms associated with the withdrawal bleed such as dysmenorrhoea or menstrual migraine and for women on enzyme-induced drugs like anticonvulsants which theoretically reduce pill efficacy.

The principle mode of action of combined hormonal contraception (CHC) is the inhibition of ovulation. Oestrogen inhibits pituitary follicle-stimulating hormone (FSH) suppressing the development of the Luteinizing hormone (LH) surge. Pills are administered for 21 days followed by a 7-day hormone-free interval (HFI). Additional properties of the contraceptives changes in cervical mucus characteristics interfering with sperm transport a possible alteration in tubal motility, endometrial atrophy and impaired uterine receptivity.

\section{ORAL CONTRACEPTIVES INDUCED HEPATOTOXICITY}

Oral contraceptives and anabolic steroids as associated with cholestasis, vascular lesions and hepatic neoplasm. Benign hepatic neoplasms are clearly associated with OCS.

\section{Cholestasis}

The frequency of cholestasis with OCS is 2.5 per 10,000 women exposed. Individuals with a previous history of the cholestasis of pregnancy are also at risk $(50 \%) .{ }^{9}$ Mild prodromal symptoms such as anorexia and nausea, followed pruritus, develop 2 to 3 months after starting OCS. $^{10}$ SAP level is moderately elevated and development of the chronic cholestasis is extremely a rare occurrence, generally recover within the days to weeks after drug cessation. ${ }^{11}$ Hormone replacement therapy (HRT) is safe in patients with liver diseases, but not recommended to jaundiced patients as the increase in bilirubin levels. So, it's necessary to monitor with liver tests.

\section{Benign neoplasm}

OCS can induce enlargement of the preexisting hemangiomas through their trophic effect on the vascular endothelium. ${ }^{12}$ Recurrences of the hemangiomas have also seen in patients with a history of previous resected lesions. A role for the estrogens in the pathogenesis of FNH is possible because these lesions occur principally in young women $(86 \%) .{ }^{14}$ In some cases liver adenomas are associated with the use of OCS. A study of over 200 patients with FNH failed to show a relationship between OCS and number of FNH lesions. ${ }^{13}$ The association between OCS and the hepatic adenomas was described approximately 30 years ago. In 1970s the increasing frequency of the neoplasm has been raised with OCS use. The long-term OCS users (>10 years) has the risk of 100fold of benign neoplasms. Patients with adenomas usually regress after OCSS are withdrawn, but surgery is required to avert possible rupture and has a definite risk of malignant transformation. ${ }^{15}$ To prevent adenomas, OCSs with lower estrogenic potency are recommended.

\section{Hepatocellular carcinoma (HCC)}

The relative risk of HCC increases with twofold among women taking OCS and has a increasing risk of sevenfold in long term users compared to age matched control. ${ }^{16,17}$ But the estrogen-related HCC is rare and less than $2 \%$ of primary liver cancer observed in western countries. In context, OCS is not an independent cause of the HCC as far importance with the chronic viral hepatitis. ${ }^{18}$

\section{CONCLUSION}

Chronic use of oral contraceptives is associated with the development of hepatic adenomas, benign tumors typically observed only in women of childbearing age and these can be resolved completely with drug withdrawal, and development of the risk factors depends upon the duration of the drug exposure. Hepatocellular carcinomas have been associated with the chronic use of anabolic, androgenic, and contraceptive steroids. Intensive researches are needed on contraceptives about the underlying mechanism of these agents produce tumors, malignancy tumors and benign adenomas. Oral contraceptives can also cause peliosis hepatis, in which weakening of sinusoidal membrane leads to the development of blood-filled sacs within the hepatic parenchyma.

\section{REFERENCES}

1. Farrel GC. Liver diseases due to environmental Toxins. In: Farrell GC, ed. Drug-Induced liver disease. Edinburgh: Churchill Livingstone, 1994:511-49.

2. Bjornsson E. Review article: Drug-induced liver injury in clinical practice. Aliment Pharmacol Ther 2010;32:3-13.

3. Ostapowicz G, Fontana RJ, Schiødt FV. Results of a prospective study of acute liver failure at 17 tertiary care centers in the United States. Ann Intern Med 2002;137:947-54.

4. Zimmerman HJ. Drug-induced liver disease. Clin Liver Dis 2000;4:73-96.

5. de Abajo FJ, Montero D, Madurga M, García Rodríguez LA. Acute and clinically relevant druginduced liver injury: a population based case-control study. Br J Clin Pharmacol 2004;58:71-80.

6. Bissell DM, Gores GJ, Laskin DL, et al. DrugInduced Liver Injury: Mechanism and test systems. Hepatology 2001;33:1009-13.

7. Dawe F, Rainford L. Contraception and sexual health 2003. A report on research using the ONS omnibus survey produced by the office for National Statistics on behalf of the Department of Health, London. Office for National Statistics, 2004.

8. Speroff L, DeCherney A. Evaluation of new generation of oral contraceptives. The Advisory 
Board for the New Progestins. Obstet Gynecol 1993;81:1034-47.

9. Czaja AJ. Current and future treatments of autoimmune hepatitis. Expert Rev Gastroenterol Hepatol 2009;3:269-91.

10. Bjornsson E, Talwalkar J, Treeprasertsuk S, et al. Drug-induced autoimmune hepatitis: clinical characteristics and prognosis. Hepatology 2010;51:2040-8.

11. Czaja AJ. Drug-Induced Autoimmune-Like Hepatitis. Dig Dis Sci 2011;56:958-76.

12. Castiella A, Lucena MI, Zapata E, et al. Autoimmune hepatitis induced by drugs. Gut 2009;58:A198.

13. Aithal GP, Watkins PB, Andrade RJ, Larrey D, Molokhia M, Takikawa $\mathrm{H}$, et al. Case definition and phenotype standardization in drug-induced liver injury. Clin Pharmacol Ther 2011;89:806-15.

14. Andrade RJ, Robles M, Lucena MI. Rechallenge in drug-induced liver injury: the attractive hazard. Expert Opin Drug Saf 2009;8:709-14.
15. Suzuki A, Brunt EM, Kleiner DE, Miquel R, Smyrk TC, Andrade RJ, et al. The use of liver biopsy evaluation in discrimination of idiopathic autoimmune hepatitis versus drug-induced liver injury. Hepatology 2011;54:931-9.

16. Goldfarb S. Sex hormones and hepatic neoplasia. Cancer Res 1976;36:2584-8.

17. Neuberger JM, Davis M, Williams R. Clinical aspects of oral contraceptive associated liver tumours. In: Davis M, Tredger JM, Williams R, editors. Drug Reactions and the Liver. London: Pitman Medical; 1981:271-83.

18. Ungo JR, Jones D, Ashkin D, Hollender ES, Bernstein D, et al. Antituberculosis drug-induced hepatotoxicity. The role of hepatitis $\mathrm{C}$ virus and the human immunodeficiency virus. Am J Respir Crit Care Med 1998;157:1871-6.

doi: 10.5455/2319-2003.ijbcp20130118

Cite this article as: Srikanth BA, Manisree V. Oral contraceptives induced hepatotoxicity. Int J Basic Clin Pharmacol 2013;2:91-3. 